\title{
Quantum Mechanics: Keeping It Real?
}

\author{
Craig Callender
}

July 23, 2020

It is well-known that when Erwin Schrödinger discovered quantum theory he wanted to interpret the quantum wavefunction $\psi$ as representing a continuous distribution of charge traveling through three-dimensional space. What is less appreciated is that Schrödinger also originally desired that his wavefunction be represented by a real-valued function and not a complex one. In some of the first papers written on quantum theory and in letters to Hendrik Lorenz and Max Planck, Schrödinger describes his progress and struggles with finding a real-valued wave equation, even as he knew the complex-valued equation that goes by his name. Eventually he discovered a completely real equation, equivalent to the Schrödinger equation, and referred to it as the "the uniform and general wave equation for the scalar field $\psi "(2020,163)$. To Planck he characterizes this breakthrough as one of "unheard of simplicity and unheard of beauty" (Przibram 1967, 16).

This paper is a kind of advertisement for exploring this form of the Schrödinger equation. Suppose we treat this real equation as another formulation of quantum theory, like the Heisenberg formulation, or even as offering a different ontology, one identifying the real part of the wave function with what J.S. Bell 2004 called a beable. Might we regard some open problems differently, and what new problems would arise? After sketching the history and some of the background, I'll illustrate how using this alternative form can be useful in understanding a problem in quantum foundations. Inspired by a recent paper by Struyve 2020, I'll show how the "real Schrödinger equation" dramatically alters a puzzle about time reversal invariance in quantum theory. My hope is that readers will find other examples like this where "keeping it real" can help.

\section{Schrödinger's Struggle for Reality ${ }^{1}$}

Schrödinger grappled with the need for quantum mechanics to use complex numbers as he was developing quantum mechanics in the Spring of 1926. In this period Schrödinger published six communications in Annalen der Physik in which he discovered his famous

\footnotetext{
${ }^{1}$ Since I wrote this section a better history of this episode has appeared in Karam 2020b, which includes examination of Schrödinger's derivation in one of his notebooks. I enthusiastically recommend Karam's paper to interested readers. My own path to this topic came via the Nobelist Yang's 1987 note, which in turn led me to Schrödinger's original papers and Chen's fascinating series of articles (1989, 1990, 1991, 1993).
} 
equation but also a real-valued version of it. He eventually abandoned the real version. However, from these six articles (which went on to become the first book of quantum mechanics) and several letters to Planck and Lorentz we know he probably did so reluctantly. ${ }^{2}$

Let's briefly recall Schrödinger's path to quantum mechanics. Schrödinger wanted deBroglie's matter waves to emerge as solutions of a wave equation. So he expected to find a second-order wave equation, one similar to what one uses in classical electromagnetism and elsewhere in physics. That equation would describe "matter waves" represented by time-dependent real-valued wave amplitudes and these waves would travel through three-dimensional space. Schrödinger's road to this equation began with the classical Hamilton-Jacobi equation. He knew that the Hamilton-Jacobi equation could be turned into a second order wave equation. Letting $\psi$ represent some "vibration process" in the atom (Schrödinger 2020, 19), he could change the former into the latter via the transformation $S=k \ln \psi$, where $k=h / 2 \pi$. His idea was that classical Hamilton-Jacobi theory would be the geometric optics limit of this new wave theory of matter.

In his first communication Schrödinger had already discovered what we now consider the time-independent version of the Schrodinger equation:

$$
\Delta \psi+\frac{8 \pi^{2}}{h^{2}}(E-V) \psi=0
$$

where $E$ is the total energy, $V$ the potential energy and $h$ Planck's constant. This equation, however, was not satisfactory, as it "suffers from the disadvantage that it expresses the law of variation of the "mechanical field scalar" $\psi$ neither uniformly nor generally" (Schrödinger 2020, 162). In an impressively long and detailed letter the 72 year old H.A. Lorentz points out various deficiencies of having the equation apply only to a specific energy. It means, for instance, that there will be "as many wave problems as there are energy values E" (Przibram 1967, 49). Lorentz urged him to eliminate $E$ from the equation in favor of time derivatives of $\psi$ - that way Schrödinger could also handle non-trivial time dependent phenomena. Schrödinger agreed. Fixing these two issues would mean finding a wave equation that was more general and more uniform.

We also know that he wanted this equation to be real-valued. In his third communication he had introduced a complex wavefunction, intending one to use only the real part. In his reply to Lorenz on June 6, he commented that

What is unpleasant here, and indeed directly to be objected to, is the use of complex numbers. $\psi$ is surely a fundamentally real function and therefore in Eq. 35 of my third paper... I should be good and write a cosine instead of the exponential, and ask myself: is it possible in addition to define the imaginary part unambiguously without reference to the whole behavior of the quantity

\footnotetext{
${ }^{2}$ For references to these six communications I'll use a new edition of the Collected Papers on Wave Mechanics (Schrödinger 2020), which is a translation from the second German edition by Blacki and Son Limited, London and Glasgow 1928. This collection also contains three additional papers from 1927. For the letters with Lorentz and Planck, I'll refer to their English translations in Przibram 1967.
} 
in time, but rather referring only to the real quantity itself and its time and space derivatives at the point in question (Przibram 1967, 62-63).

The wave equation he desired would be general, uniform and real.

He achieves all three goals by letting the real part of a complex wavefunction have a (periodic) time dependence. He differentiates it twice with respect to time to obtain

$$
\frac{\partial^{2} \psi}{\partial t^{2}}=-\frac{4 \pi^{2} E^{2}}{h^{2}} \psi
$$

, or in terms of a Hamiltonian $H, H \psi=E \psi$. Plugging this into the above timeindependent equation yields

$$
\left(\nabla^{2}-\frac{8 \pi^{2}}{h^{2}} V\right)^{2} \psi+\frac{16 \pi^{2}}{h^{2}} \frac{\partial^{2} \psi}{\partial t^{2}}=0
$$

which can be compactly expressed via $\hbar$ as

$$
\hbar^{2} \frac{\partial^{2} \psi}{\partial t^{2}}=-H^{2} \psi
$$

In his fourth communication he proclaims his version of (1) to be "evidently the uniform and general equation for the field scalar $\psi$ " (Schrödinger 2020, 163). This equation is the first published time-dependent Schrödinger equation. It meets all of his desiderata: it is real-valued, general, and time-dependent. ${ }^{3}$ It is real in that both $\psi$ is a real-valued function and in that equation (1) has no imaginary components to it.

Equation (1) is second-order in time like a wave equation, but fourth-order due to the spatial derivatives. That makes it more complicated than the equation with which we're familiar (which is derived by inserting the first rather than second derivative of a complex rather than real wavefunction into the time-independent equation). That additional complexity by itself probably didn't bother Schrödinger too much. The historians Mehra and Rechenberg 1987 write that he "was not afraid of mathematical complications of higher order equations; the methods of their solution were well known in principle" (788). In his work he regularly entertained equations of high order. Indeed, in his letter to Planck, after introducing (1) he immediately notes that it is "completely the same type as the equation for a vibrating plate" (Przibram 1967, 17) and he reproduces this equation in a footnote in his fourth communication. ${ }^{4}$

Schrödinger is evidently relieved at having discovered (1). He writes on June 11 to Max Planck

By the way, during the last few days another heavy stone has been rolled away from my heart....I had considerable anxiety over it...But it all resolved itself with unheard of simplicity and unheard of beauty; it all came out exactly as one would have it, quite straightforwardly, quite by itself and without forcing. (Przibram 1967, 16)

\footnotetext{
${ }^{3}$ See Karam 2020b for discussion of Schrödinger's derivation of this equation in his notebook.

${ }^{4}$ The equation for a classical vibrating plate is of the form $\nabla^{2} \nabla^{2} \psi+\partial^{2} u / \partial t^{2}=0$.
} 
What he is referring to is his discovery of (1), which he describes as "the true wave equation which is of fourth order in the coordinates" (17). His goal of a wave equation that is general, uniform and real was achieved.

Not long after, however, he became worried about the introduction of time varying potentials. Terms such as $\partial V / \partial t$ demanded "caution" (Schrödinger 2020, 164). He noticed that another path was possible, one "extraordinarily much simpler for computational purposes" (Przibram 1967, x). This equation, of course, was the second-order equation that we now refer to as the Schrödinger equation. The price of this simplicity was the introduction of complexity. By the time of his sixth communication he decided to use the complex equation. At this point it's clear that he regarded his namesake as a kind of surrogate for the entirely real fourth-order equation (1). At the very end of this paper he writes:

There is undoubtedly a certain crudeness at the moment in the use of a complex wave function. If it were fundamentally inevitable and would not be a mere simplification of the calculation, it would mean that there are basically two wave functions that only give information about the state of the system together. This somewhat unsympathetic conclusion, I believe, allows the much more sympathetic interpretation that the state of the system is given by a real function and its derivation over time.

So although he used the complex equation at the end of this important period, he viewed it as a kind of convenient substitute for a real function and its time derivatives.

It is not known how long Schrödinger's belief in the "reality" of quantum mechanics persisted nor precisely why he abandoned the real equation. Chen 1989 holds that Schrödinger's difficulties in finding a non-conservative version was a major hurdle (but shows how it is surmountable); Karam 2020b suggests that the lack of a physical interpretation for the real part may have been a problem and that the growing use of $\psi^{*} \psi$ as the charge density, which is real, may have satisfied him. Recall that Schrödinger never accepted the statistical interpretation of the wavefunction. Also, note that his real-valued wavefunction still "lives" in high-dimensional configuration space, just like the complex-valued wavefunction; so the move to real values doesn't by itself help one interpret it as an object or field in three-dimensional space. To me it's plausible that the combination of issues caused him to put the problem aside. What he originally wanted was hard to use, difficult to interpret, and to some extent almost achieved because $\psi^{*} \psi$ was real. ${ }^{5}$

In any case, almost exactly a year (June 10,1927) later Schrödinger writes in a footnote that

The wave function has to be regarded as essentially complex (Schrödinger 2020, 216).

\footnotetext{
${ }^{5}$ As Yang 1987 notes, it's interesting in this context to recall that Born's original expression of the probability density occurred in a footnote (in a paper received June 21, 1926) and left off the now familiar absolute value symbols. The reason? Born used real wavefunctions for both his incoming and scattered wavefunctions.
} 
However, he doesn't explain what he means by "essentially" here. There are certainly many plausible interpretations of this statement that are consistent with his earlier position. The physicist Chen, who has done the most to investigate this real Schrödinger equation, reports that V.F. Weisskopf wrote to him that "Schrödinger persisted in his belief in the real wave function until his last days" (Chen 1990). Minus the context, this too can be interpreted various ways.

That said, as late as 1952 Schrödinger wrote a note in Nature pointing out that, contrary to "widespread belief," charged fields do not need to be represented by complex numbers. Schrödinger shows that by a change of gauge the wavefunction used in the Klein-Gordon-Maxwell equations of motion can be made real (Schrödinger 1952). He finds it interesting that in this real formulation the electromagnetic potential becomes proportional to the current, just as in Dirac's recently proposed theory (for more recent work on this, see Akhmeteli 2011). That Schrödinger was still considering real wavefunctions just nine years prior to his death is suggestive of Chen's claim, although hardly definitive.

While we will likely never know how long Schrödinger's belief in a real wavefunction persisted, we can appreciate that this desire to keep quantum mechanics real was quite natural given the time period. He was of course accustomed to using complex functions and equations from classical mechanics. As his research evolved he found himself increasingly using complex numbers, especially when he was confronted with Heisenberg's theory. But in the physics he studied prior to the development of quantum mechanics, ultimately one always connected the real parts of complex equations to experiment, e.g., as in linear circuit theory. All of the physics to date suggested the imaginary was always used for convenience but ultimately dispensable.

\section{Schrödinger's Real Equation}

While we are often taught that in quantum mechanics the use of imaginary numbers is essential, that is in fact not true. ${ }^{6}$ One can represent what quantum theory has to say using real numbers, complex numbers and even quaternions, and there are plenty of formulations of the theory that are entirely real. That said, the above may be surprising for it's an elementary mathematical fact that the real part of a complex number underdetermines the complex number of which it is a part. The real part is leaving out "half" the information of the complex function. So it's worth briefly explaining how there can be a real equation with a real-valued wavefunction.

While there are some tricky mathematical details, the core idea is easy to understand. As is well-known, the complex Schrödinger equation is equivalent to two coupled differential equations for two real variables (Bohm 1951). The present idea simply uses this coupling to put everything in terms of one variable. The two equations are rewritten in terms of one variable and its time derivative.

The complex Schrödinger equation is

\footnotetext{
${ }^{6}$ Karma 2020a surveys some interesting rationales for the complexity of quantum mechanics.
} 


$$
i \frac{\partial \psi}{\partial t}=H_{t} \psi
$$

where $\psi$ is complex, $\hbar=1$, and $H_{t} \equiv\left((1 / 2 m) \nabla^{2}-V(x)\right)$. Dub $u(x, t)$ and $v(x, t)$ the real and imaginary parts of $\psi$, respectively, i.e., $\psi(x, t)=u(x, t)+i v(x, t)$. Inserting this into the complex equation (2) and separating yields two real equations

$$
\begin{gathered}
\frac{\partial u}{\partial t}=H_{t} v \\
\frac{\partial v}{\partial t}=-H_{t} u
\end{gathered}
$$

Note, crucially, that the two equations are coupled.

Using this coupling, we recognize the possibility of expressing everything in terms of one equation of $u$ and $\partial u / \partial t$. One can try to isolate $v$ by integrating with respect to time. This will bring out an arbitrary function, which is presumably what bothered Schrödinger ("integration with respect to time would involve an undetermined wave function. I do not know yet whether this can be fixed in a rational way," (Przibram 1967, X)). One can answer this problem, but another method is to find a unique inverse operator $\left(H_{t}\right)^{-1}$ of $H_{t}$ in (4), so that

$$
v=\left(H_{t}\right)^{-1} \frac{\partial u}{\partial t}
$$

. In the energy representation this operator is $\left(H_{t}\right)^{-1}=\left[\hbar / E_{i}(t)\right] \delta_{i j}$ (Chen 1989, eq. 6). ${ }^{7}$ Using this relationship allows one to write everything in terms of $u$.

Note that an analogous move is not possible in classical mechanics. Although it is not widely recognized, classical mechanics can be described by a complex first-order but nonlinear "classical Schrödinger" equation (Rosen 1964, Schiller 1962, Schleich et al 2013). One can try a similar trick and insert a complex wavefunction into this equation and separate real and imaginary parts. When one does, however, the two equations are not coupled as in quantum theory, eliminating the possibility of exploiting the coupling to eliminate the second real-valued function.

Recall that Schrödinger had concerns about the extension to non-conservative cases. Sparing the details, Chen 1989 discovered the equation Schrödinger sought,

$$
\frac{\partial^{2} u}{\partial t^{2}}=-H_{t}^{2} u+\frac{\partial V}{\partial t} H_{t}^{-1} \frac{\partial u}{\partial t}
$$

where everything is written explicitly in terms of $u$ and its time derivative. When $V$ doesn't depend on time (5) reduces to the simpler (1).

Equation (1) is a single equation and entirely real: neither $u$ nor the equation contains an $i$. (1) looks a lot like a classical wave equation. Like such equations, it is secondorder in time and takes Cauchy initial data, $u$ and $\partial u / \partial t$. Unlike classical wave equations,

\footnotetext{
${ }^{7}$ Note that $v$ 's dependence upon $u$ is apparently nonlocal. See Chen 1989 for the concern and Chen 1991 for explanation.
} 
however, it is fourth order due to the spatial derivatives (in the $H^{2}$ term). On this picture, the real part of the wavefunction is roughly like the configuration variable and the imaginary part is like its velocity. ${ }^{8}$

\section{Discussion}

Schrödinger saw that he had essentially three choices in how to represent his theory.

Use the second-order complex equation (2) for which he is known.

Use two coupled real equations, such as $(3,4)$ above.

Use the fourth-order real equation (1).

For option B I write "such as" because there are more illuminating real equations one can use, as Schrödinger knew. In particular, a more natural version of this strategy that fits his path of discovery would have been to pick two different real equations, a modified Hamilton-Jacobi type equation and a Van Vleck continuity equation. This form of quantum mechanics was used by Bohm 1952 in his derivation of the de-Broglie-Bohm interpretation of quantum mechanics and is nicely discussed by both Holland 1998 and Schleich et al 2013. These two coupled equations are derived by expressing the complex wavefunction in polar coordinates as $\psi=A e^{i S}$, where the amplitude $A$ and phase $S$ are real-valued, and then separating into real and imaginary parts.

Two initial values are necessary in all three presentations. The first requires the real and imaginary parts of the complex wavefunction; the second requires two real functions; and the third requires one real function and its temporal derivative. The $i$ in (2) enforces the coupling between these two real functions-without it the two functions would decouple and we'd have too much freedom, as we could then specify $u, \partial u / \partial t, v$ and $\partial v / \partial t$ (Bohm $1951,86)$. So by going complex both in the wavefunction and the wave equation one eliminates two types of initial data. That coupling, we saw, is then used to get the single real wave equation.

What should we make of this "new" option? One can imagine what we might call weak and strong interpretations. On the weak interpretation, we regard Option C as simply another way of expressing the same theory. Just as Newton's second law can be written as $F=m a$, or $F=d p / d t$, or $F=d(m v) / d t$, or $F=-\nabla V(x)$, so too Schrödinger's theory can be written many ways. All three options pick out the same models of the physical universe, so one is not right and the others wrong. Our selection is instead dictated by so-called "pragmatic" virtues, such as ease of use, fruitfulness, elegance, context, and purpose. Like Holland 1998 and Schleich et al 2013, I often find option B (in its modified Hamilton-Jacobi form) preferable in pedagogical contexts. It provides insight

\footnotetext{
${ }^{8}$ How a complex wavefunction is split into real and imaginary parts is to some extent arbitrary. This arbitrariness is a kind of gauge invariance. Let $\psi(x, t)=u(x, t)+i v(x, t)$. Then if $u, v$ satisfy equations (3) and (4) above, then $u^{\prime}$ and $v^{\prime}$ will satisfy the primed counterparts of these equations if $u=u^{\prime} \cos \theta-v^{\prime} \sin \theta$ and $v=v^{\prime} \cos \theta-u^{\prime} \sin \theta$. Chen 1990 shows that this transformation is independent of the Hamiltonian of the system.
} 
into the historical origin of quantum mechanics, Schrödinger's discovery, the classical-toquantum correspondence, and the deBroglie-Bohm interpretation. Confronting option $\mathrm{C}$, one question we might have is whether there are contexts where this form is similarly useful. I'll give an example where I think it is in a moment.

On this weak interpretation, I think it's fair to say that the choice Schrödinger and history made excels. Equation 2 of option A is a single compact expression of low order, unlike the equations of options B and C. It is likely the lowest order single equation that can characterize the set of worlds allowed by non-relativistic quantum mechanics (for arguments along these lines, see Bohm 1951). Furthermore, if we look past the choice of wave equation to the full theory, we will find that the extraction of probabilities from the wave equation (2) is incredibly elegant and easy via Born's rule; by contrast, it will be comparatively messy or artificial via option $\mathrm{C}$.

We can contemplate a stronger interpretation, one that Schrödinger probably initially entertained. So far I have said nothing about the interpretation of quantum mechanics. An interpretation is a mapping between the representation and the world. In the context of quantum mechanics, it's common to refer to the ontology of a theory as a beable. The question then arises as to what the beables of quantum mechanics are. Do they include the wavefunction? More-such as GRW mass density or Bohmian particles? And if the wavefunction, we now see that we have a new option - adopting the position that only the real part of the wavefunction is a beable. Call this $\operatorname{Re}(\psi)$ Realism. According to $\operatorname{Re}(\psi)$ Realism, the real-valued wave represents what is real.

I think it's plausible that Schrödinger was a $\operatorname{Re}(\psi)$ Realist, at least in June 1926. As has been chronicled many times elsewhere, we know that Schrödinger held that his wave equation described a genuine field in the world. He did not share Heisenberg's positivist inclinations about ontology. He also felt that the use of imaginary numbers in quantum theory would turn out to be understood in the way in which he was familiar classically, namely, as dispensable. But note also that classically one never regards the imaginary parts of complex representations as representing the ontology. It's not just that classical mechanics associates experimental outcomes with the real parts of complex representations (if used), it also associates the ontology of particles or fields with the real parts. The complex representation, for Schrödinger, represented a real field described by the real part of the wavefunction and its time derivative.

I'm not advocating such a position here. I note, however, that it may be attractive to some even today. Advancing the so-called "multi-field" interpretation of the wavefunction, Romano 2020 opts for path B above in part because the two fields it posits are real-valued. The real-valuedness makes it easier, he thinks, to understand how to relate these two real fields to measurement outcomes. Path $\mathrm{C}$ arguably provides the same benefit but in a more parsimonious manner, positing one real-valued field as opposed to two. A similar benefit may appear in attempts to "nominalize" quantum theory, such as Chen 2018. Something like path $\mathrm{C}$ also appears in the reduced phase space formulation of Schrödinger theory and may be helpful in considering possible beables for Bohmian field theory (Struyve 2010).

In what follows I'll describe one benefit of path C. This can be interpreted in a strong or weak way, as a consideration in favor of $\operatorname{Re}(\psi)$ Realism or as simply a pedagogical 
insight due to representing quantum theory via path C.

\section{The Puzzle of Time Reversal Invariance in Quantum Theory}

Quantum mechanics is widely proclaimed to be time reversal invariant. Yet this is a very puzzling claim. Let us assume that we use a time reversal invariant Hamiltonian such as $H=p^{2} / 2 m+V(x)$. Then the Schrödinger equation is not time reversal invariant in the usual sense (Albert 2000, Callender 2000). Normally one associates time reversal in classical spacetimes with the physical operation corresponding to a temporal reflection about a spatial axis: the past is swapped for the future. Then, in natural coordinates, time reversal corresponds to the action $T: t \rightarrow-t$. Yet if we apply this operation to the Schrödinger equation, one immediately sees that it is not time reversal invariant. Temporal reflection brings out a negative sign in front of the equation because (2) is first-order in time and nothing else changes. Thus it is typically the case that if $\psi(t)$ is a solution then $\psi(-t)$ is not.

To drive the point home, note that the heat equation has the form

$$
\frac{\partial \psi}{\partial t}=\kappa \nabla^{2} \psi
$$

where $\kappa$ is a real-valued coefficient. The heat equation is the paradigmatic non-time reversal invariant equation-it describes the irreversible diffusion of heat through a medium. And as we would expect of such an equation, we can readily see that if $\psi(t)$ is a solution then $\psi(-t)$ is not. The equation is not invariant under a temporal reflection. The only relevant formal difference between (6) and the Schrodinger equation (2) is that (2) has an $i$ in it. But why should an $i$ - which is just a number - matter? A physical operation in spacetime, a temporal reflection, should not affect dimensionless numbers.

Quantum textbooks sometimes address this point and claim that in quantum mechanics time reversal invariance is to be given by two operations, a temporal reflection and the operation of complex conjugation $K: \psi \rightarrow \psi *$. This idea can be traced back to Wigner 1936. With this understanding quantum mechanics is indeed time reversal invariant. But it's also clear that this combined operation, however one refers to it, is different than merely a temporal reflection. It is also a very strange symmetry, as it is the only one in quantum theory represented by an anti-unitary operator. Its introduction demands some explanation and justification. After all, what physical symmetry is represented by complex conjugation, and what does it have to do with time reversal?

Some distinguished books find this task very difficult. Roman 1950, for example, writes that "we should like, even if only by some artifice, to achieve full covariance [time reversal invariance]" (1950, 266). Sachs 1987 offers one of the more careful treatments one can find and plausibly insists that time reversal should leave the kinematical structure of the theory intact; however, a crucial part of his argument - like that of many others is that complex conjugation is necessary if time reversal is to reverse momentum and spin. That's true but it just pushes the question back, for momentum and spin are both 
spatial in quantum mechanics. Momentum is a spatial derivative and spin lacks timedependence and is sometimes understood as a form of space quantization. Neither gets reversed by taking a temporal reflection.

Others (e.g., Roberts 2020) argue that the combined operations of temporal reflection and complex conjugation better merit the denomination "time reversal invariance" than temporal reflection does. The idea is that one can place various conditions on what one wants from time reversal and then show that the combined operation fits these conditions and temporal reflections alone do not. That's fine as far as it goes, but why does what we call the operation matter? Call it what you like, Wigner's reversal is different from a temporal reflection and taking the complex conjugation of a state doesn't follow by logic or definition alone from a temporal reflection. Classical mechanics is invariant under temporal reflections (so long as friction and other non-conservative forces aren't included). Quantum theory a la the Schrödinger equation (2) is not if we treat like for like. We can agree to call the combined operations "time reversal invariance" but that doesn't make the problem go away.

My own best effort (Callender 2000) used the classical correspondence and Ehrenfest's theorem to tie Wigner reversal to classical time reversal invariance. This approach, I think, provides some insight and explains why Wigner's transformation is so important. But I don't think it gets to the heart of the puzzle, which is that I've never seen an argument that links by logic or definition alone temporal reflections to complex conjugation.

Schrödinger's real realism offers insight here. Although he does not tie it to Schrödinger, Struyve 2020 considers altering the ontology of classical electromagnetism and quantum theory in various ways to see what effect this has on time reversal invariance. Because a symmetry leaves the ontology of a theory intact, it is natural to expect the fate of symmetries to hang on the choice of ontology (and perhaps vice versa, inasmuch as symmetries may play a role in the selection of ontology). Struyve shows that this is indeed so for time reversal invariance if one adopts what I'm calling $\operatorname{Re}(\psi)$ Realism.

Suppose that the ontology of quantum theory is given by the real part of the wavefunction and the main governing law of nature is best formulated as Schrödinger's real equation, (1) above. Perform a temporal reflection on (1). Because it is second order in time and nothing else changes (as we'll see, and assuming, as usual, a time reversal invariant Hamiltonian), it is manifestly time reversal invariant. Use the complex wavefunction and (2), by contrast, and the theory is not invariant under a temporal reflection. There is no paradox here because the ontology is not the same in the way we're imagining things. This is an interesting example of Struyve's point that the symmetry changes with the ontology.

Can this case provide insight into our puzzle? Yes. Suppose we introduced the above puzzle about time reversal to Schrödinger in June 1926. Let's call him Real Schrödinger. How might Real Schrödinger understand and react to our problem?

Recall that Real Schrödinger would view the introduction of $i$ into the wave equation as the price one pays for going lower order in type of differential equation and thereby getting something "extraordinarily much simpler for computational purposes." The $i$ plays an absolutely central role in allowing (2) to express the same theory as $(3,4)$ and also (1). So when considering time reversal invariance, Real Schrödinger would want to 
"unwind" the transformation of his theory into a complex representation and understand time reversal in terms the real formalism and $u$ and $\partial u / \partial t$. Looking at that formulation, we've already seen that

$$
v=\left(H_{t}\right)^{-1} \frac{\partial u}{\partial t}
$$

holds, i.e., that the imaginary part of the wavefunction contains the information about the time dependence of the real part. Here, Real Schrödinger will think, is my time dependence of $u$. The imaginary part is roughly like the velocity of the real part-due to the presence of $\left(H_{t}\right)^{-1}$ the imaginary part is not fully analogous to velocity. The important point is that $\left(H_{t}\right)^{-1}$ will not flip under time reversal. Hence by definition alone $v$ must flip under time reversal. ${ }^{9}$

Looking at the complex formulation, Real Schrödinger will see that this time dependence has been disguised by the complex clothing. He would know that he needs to invert $v$. In the complex formulation the way to do this is to change $\psi=u+i v$ to $\psi=u-i v$ or vice versa. So he would end up changing $\psi$ to $\psi^{*}$. He would do this not because a dimensionless quantity like $i$ mysteriously flips under time reversal, but simply because $v$ is a disguised first derivative in time. Put picturesquely, envision the wavefunction as expressed in a complex space spanned by its real and imaginary axes. Changing the sign of the imaginary part of the wavefunction will force the real part to rotate in the opposite direction.

This solves our puzzle. Seen through the prism of the real formalism, we can understand why complex conjugation is connected to temporal reflection. Formulated in the real theory version, a temporal reflection essentially reverses the velocities. But now if we express the theory in its complex formulation, we see that the definitional counterpart of reversing the velocities is taking the complex conjugate of the full wavefunction. Complex conjugation of the full wavefunction is just another way of expressing a temporal reflection of the real part of the wavefunction. Although the Schrödinger equation and the heat equation look almost the same, that similarity turns out to be superficial. The complex wavefunction interacting with the $i$ in the general wave equation masks a time dependence that has no counterpart in the heat equation.

I emphasize that I am not saying that we either know quantum theory is or isn't time reversal invariant nor am I advocating for a particular ontology. The choice of correct ontology and its symmetries is a large one hanging on many factors. What I am saying is that this real formalism provides greater insight into the conventional claim that quantum theory is time reversal invariant than I've seen elsewhere. For me, this explanation provides a far more satisfying justification for thinking quantum theory time reversal invariant than I've previously encountered. It doesn't demand that we call one symmetry another because that is intuitive, nor does it rely on arguments that

${ }^{9}(7)$ can be expressed as $v=\int G \frac{\partial u}{\partial t} d x^{\prime}$ where $G$ is the Green's function of the time-independent Schrödinger equation (Chen 1991, eq 7). What is crucial to our discussion is that the Green's function for the time independent Schrödinger equation doesn't flip under time reversal. For a free particle in one dimension, for example, $G=G\left(x, x^{\prime}\right)=\left(\left|x-x^{\prime}\right|\right)^{-1}$, which is unaffected by a temporal reflection. 
don't survive much scrutiny (that momentum must alter sign, despite being a spatial derivative), nor does it merely inform us that Wigner reversal is important. Just as the second path via two real wave equations can be illuminating when discussing the classical correspondence and much else, path $\mathrm{C}$ can teach us about time reversal invariance.

In fact it is possible that looking at time in quantum mechanics through this perspective will be useful elsewhere too. Could it provide new insights into how to understand stationary states? The CPT theorem? We'll leave these questions for future work.

\section{Conclusion}

This paper has been an introduction to and advertisement of Schrödinger's real wave equation, the first published time dependent Schrödinger equation. I introduced two ways of interpreting this equation, one weak, one strong. And I have shown how this equation is not merely a historical curiosity. I mentioned how it may provide an attractive option for a wavefunction realist and illustrated the insight it provides into quantum time reversal invariance. It is my hope that other examples like this will be forthcoming. ${ }^{10}$

\section{References}

[1] Akhmeteli, A. 2011. One Real Function Instead of the Dirac Spinor Function. Journal of Mathematical Physics 52:8, 082303.

[2] Albert, D. Z. 2000. Time and Chance, Harvard University Press.

[3] Bell, J. S. 2004. Speakable and Unspeakable in Quantum Mechanics: Collected Papers on Quantum Philosophy. Cambridge: Cambridge University Press.

[4] Bohm, D. 1951. Quantum Theory. Prentice-Hall.

[5] Bohm, D. 1952. A Suggested Interpretation of the Quantum Theory in Terms of "Hidden" Variables: I and II, Physical Review 85(2), 166-193.

[6] Callender, C. 2000. Is Time 'Handed' in a Quantum World?, Proceedings of the Aristotelian Society, Aristotelian Society, 247-269.

[7] Chen, E.K. 2018. The Intrinsic Structure of Quantum Mechanics. arXiv:1810.06551 [quant-ph].

[8] Chen, R. L. W. 1989. Derivation of the Real Form of Schrödinger's Equation for a Nonconservative System and the Unique Relation Between $\operatorname{Re}(\psi)$ and $\operatorname{Im}(\psi)$. Journal of Mathematical Physics 30, 83-86.

[9] Chen, R. L. W. 1990. The Real Wave Function as an integral Part of Schrödinger's Basic View on Quantum Mechanics. Physica B167, 183-184.

\footnotetext{
${ }^{10}$ Thanks for comments and discussion to Jeff Barrett, John Dougherty, Ricardo Karam, Davide Romano, Chip Sebens, Paul Skokowski, and Ward Struyve.
} 
[10] Chen, R. L. W. 1993. Schrödinger's Real Wave Equation (Continued): Why Did He Fail to Derive it in its General Form? Physica B190, 256-258.

[11] Chen, R. L. W. 1991. Concerning the Gauge Invariance and the Apparent Nonlocality of the Real Form of Schrödinger's Equation. Journal of Mathematical Physics 32,464 .

[12] Courant, R.,and Hilbert, D., 1924, Merhoden der Marhematischen Physik I, Berlin: Springer.

[13] Holland, P.R. 1993. The Quantum Theory of Motion: An Account of the de BroglieBohm Causal Interpretation of Quantum Mechanics, Cambridge University Press, Cambridge.

[14] Karam, R. 2020a. Why are Complex Numbers Needed in Quantum Mechanics? Some Answers for the Introductory Level. American Journal of Physics 88, 39-45.

[15] Karam, R. 2020b. Schrödinger's Original Struggles with a Complex Wave Function. American Journal of Physics 88, 433-438.

[16] Mehra, J. and Rechenberg, H. 1987. The Historical Development of Quantum Theory. Volume V: Erwin Schrödinger and the Rise of Wave Mechanics, Part II. New York/Heidelberg, Springer-Verlag.

[17] Przibram, K. (ed.) 1967. Letters on Wave Mechanics: Schrodinger, Planck, Einstein, Lorentz. NY: Philosophical Library Inc.

[18] Roberts, B. 2020. Time Reversal. The Routledge Companion to the Philosophy of Physics, Eleanor Knox and Alistair Wilson (eds).

[19] Roman, P. 1960. Theory of Elementary Particles. NY: Interscience Publishers.

[20] Romano, D. 2020. Multi-field and Bohm's theory. Synthese, forthcoming.

[21] Rosen, N. 1964. The Relation Between Classical and Quantum Mechanics. American Journal of Physics 32, 597-600.

[22] Sachs, R. G. 1987. The Physics of Time Reversal, Chicago: University of Chicago Press.

[23] Schiller, R. 1962. Quasi-classical Theory of the Non-spinning Electron. Physical Review $125,1100-1108$.

[24] Schleich, W. P., Greenberger, D. M., Kobe, D. H., \& Scully, M. O. 2013. Schrödinger Equation Revisited. Proceedings of the National Academy of Sciences of the United States of America 110, 5374-5379.

[25] Schrödinger, E. 1952. Dirac's New Electrodynamics. Nature (London) 169, 538. 
[26] Schrödinger, E. 2020. Collected Papers on Wave Mechanics, with an introduction by Valia Allori. Translated from the second German edition (Blacki and Son Limited, London and Glasgow 1928).

[27] Struyve, W. 2010. Pilot-wave Theory and Quantum Fields. Reports on Progress in Physics 73, 106001.

[28] Struyve, W. 2020. Time-reversal Invariance and Ontology. Forthcoming.

[29] Yang, C.N. 1987. Square Root of Minus One, Complex Phases and Erwin Schrödinger, In Schrödinger: Centenary Celebration of a Polymath, edited by C. W. Kilmister (Cambridge U. P., Cambridge), 53-64. 